\title{
Quantitative Analysis of Energy-Dispersive Spectra Acquired with Large Solid Angle Detectors
}

\author{
Ralf Terborg $^{1}$, Meiken Falke ${ }^{1}$, Tanja Mohr-Westheide ${ }^{2,3}$, Tobias Salge ${ }^{4}$ \\ ${ }^{1}$ Bruker Nano GmbH, Am Studio 2D, 12489 Berlin, Germany \\ ${ }^{2}$ Museum für Naturkunde Berlin, 10115 Berlin, Germany \\ ${ }^{3}$ Freie Universität Berlin, Institut für Geologische Wissenschaften, 12249 Berlin, Germany \\ ${ }^{4}$ Imaging and Analysis Centre, Natural History Museum, London, UK
}

During the last years Silicon Drift Detectors (SDDs) for energy-dispersive spectrometers (EDS) with larger and larger areas have been released by the manufacturers. While SDDs with an active area of e.g. $10 \mathrm{~mm}^{2}$ were widely in use a decade ago modern detectors can have areas over $100 \mathrm{~mm}^{2}$.

One of the most important parameter for performance is the solid angle as this mainly defines the collection efficiency. Therefore, not only the active area but also the distance of the detector front to the sample is relevant $[1,2]$. Geometrical changes in the detector shape where the outer dimensions have been optimized to get closer to the sample were implemented as well. Solid angles in the range of $0.1 \mathrm{sr}$ can be reached with such an optimized setup.

In addition, unconventional geometries like an annular quad detector (XFlash FlatQuad), which is placed between pole piece and sample, have been developed which reach a solid angle of more than $1.1 \mathrm{sr},[3]$, fig $1 \mathrm{a}$.

When using these large solid angle detectors for quantitative analysis not only X-rays from the nominal take-off angle (TOA) are collected. Instead an integrated X-ray signal within a certain range of TOAs is recorded. For a conventional detector with a solid angle of $0.1 \mathrm{sr}$ the TOA can vary by $\pm 10^{\circ}$, so for a nominal TOA of $\psi=35^{\circ}$ ranges of $25^{\circ}$ to $45^{\circ}$ apply, fig. $1 \mathrm{~b}$.

Because the TOA is important for the absorption of X-rays in the sample and in the detector layers the contribution of the X-rays from various TOAs need to be taken into account.

For standardbased analysis these effects mostly cancel out when unknown and standard are acquired with the same setup. But for standardless analysis this effect might influence the results.

The different amount of absorption for different TOAs leads to changes in the peak intensities, especially for low-energy lines $(<1 \mathrm{keV})$, and therefore influence the results of quantitative analysis of such spectra.

To evaluate this effect and the uncertainties, results from a simple quantification method are compared to full calculations integrating the results considering the full TOA range. Further applications will be discussed in the presentation.

\section{References:}

[1] M Procop et al., Microsc. Microanal. 22 (2016), p. 1360

[2] NJ Zaluzec, Microsc. Microanal. 20 (2014), p. 1318

[3] R Terborg et al., Microsc. Today 25 (2017), p. 30 

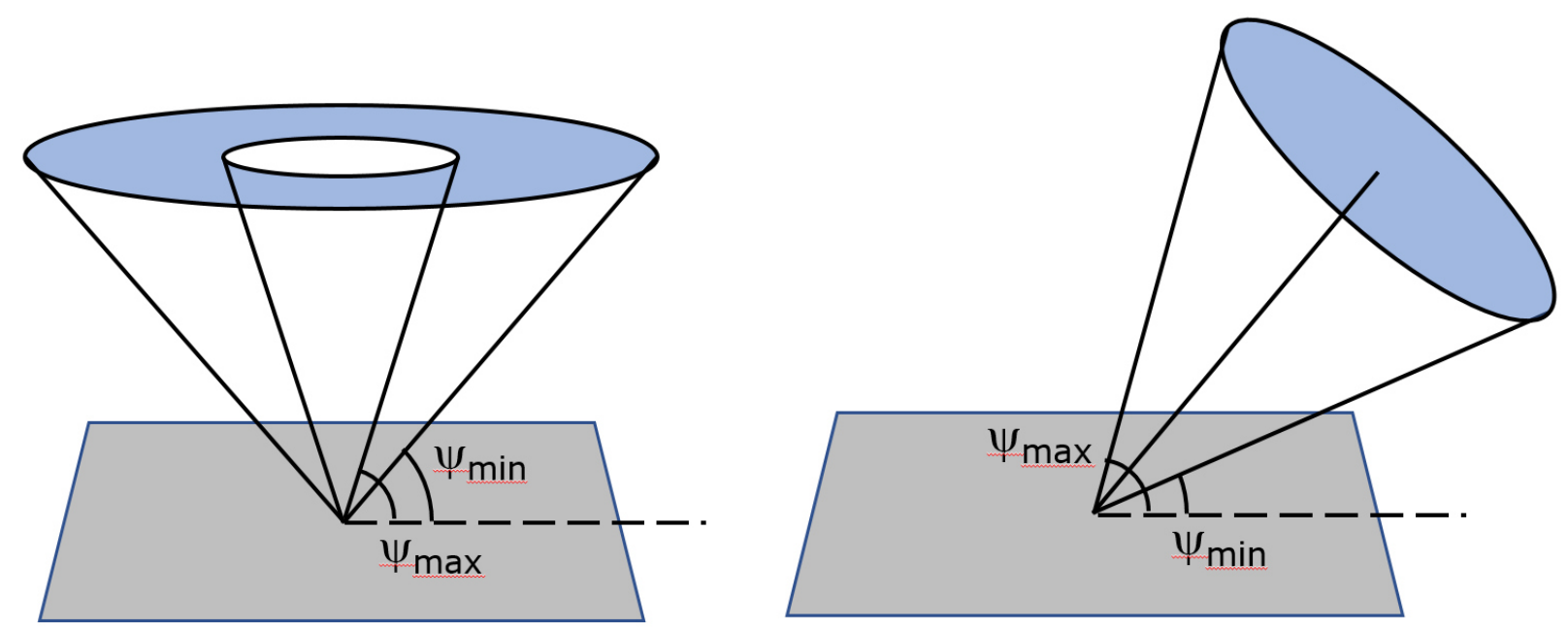

Figure 1. Take-off angle range for an annular (a) and a conventional circular detector (b).

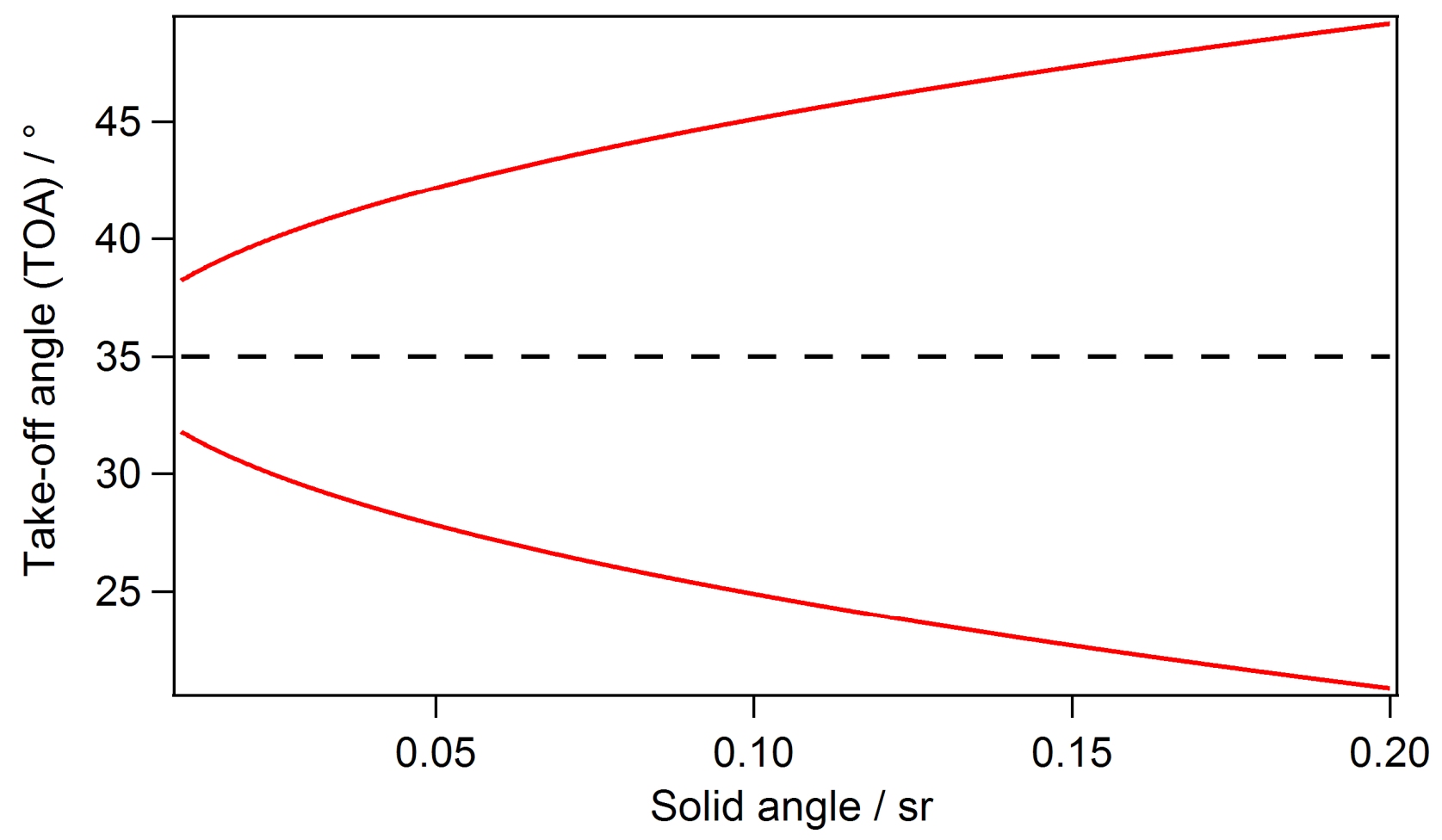

Figure 2. Take-off angle range and minimum and maximum TOA for a circular detector with a nominal TOA of $35^{\circ}$. For a detector covering a solid angle of $0.1 \mathrm{sr}$ the TOA varies by $\pm 10^{\circ}$. 